\title{
Venous Sinus Stenosis Causing Isolated Pulsatile Tinnitus
}

\author{
Miguel Quintas-Neves (D), Eduardo Freitas, José Manuel Amorim, Jaime Rocha, \\ João Pinho
}

Keywords: Pulsatile tinnitus, Venous sinus

doi:10.1017/cjn.2019.73

Can J Neurol Sci. 2019; 46: 591-592

A 61-year-old man was referred to the neurology clinic due to a persistent, disabling, right-sided pulsatile tinnitus (PT), which he noticed 4 years before and had recently worsened. The tinnitus ameliorated with manual pressure over the ipsilateral cervical region and cervical flexion to the right side. With the exception of an audible high-pitch bruit below the right mastoid process, the neurological examination was unremarkable. After careful evaluation, computed tomography angiography and magnetic resonance imaging showed a stenosis in the right lateral venous sinus (Figure 1A-C). Lumbar puncture revealed an intracranial pressure of $12 \mathrm{cmH}_{2} \mathrm{O}$. The presence of a stenosis-associated pressure gradient was confirmed in digital subtraction angiography $(9 \mathrm{mmHg})$, by using a co-axial system consisting of a NeuronMax 0.88 long sheath (placed in the right internal jugular vein) and a 0.021 " microcatheter positioned on the superior sagittal sinus (Prowler Select Plus). Following multidisciplinary discussion and patient agreement, recalibration of the venous sinus was successfully performed with a nontapered X-Act stent (9 x $30 \mathrm{~mm})$ (Figure 1D-E), after progression along a microguidewire Synchro 0.014". Tinnitus resolved immediately after the procedure, and it had not recurred at the 6-month follow-up.

Only $10 \%$ of all patients with tinnitus describe it as pulsatile in nature. ${ }^{1}$ There are multiple causes for PT, namely, intracranial or cervical arterial/venous/arteriovenous abnormalities, vascularized tumors in the vicinity of the ear, intracranial hypertension, and semicircular canal dehiscence, which must be carefully searched for. ${ }^{2}$ Venous origin may be suspected when PT decreases or resolves with ipsilateral venous compression, valsalva maneuvers and rotation of the head toward the affected side, and increases with contralateral venous compression and rotation of the head to the contralateral side. Lateral venous sinus stenosis has long been recognized as a cause for isolated PT. ${ }^{3}$ Even though underlying pathophysiological mechanisms are unclear, a significant trans-stenotic pressure gradient is probably required so that when systolic arterial pulses occur, the cerebral veins and sinuses are rhythmically compressed, leading to an increased, turbulent trans-stenotic venous flow. Venous sinus stenting has gained acceptance as treatment option for patients with intracranial hypertension and venous sinus stenosis, with improved papilledema, headache, and PT outcomes. ${ }^{4}$ However, for patients with isolated PT with no intracranial hypertension, the benefit of stenting and the peri- and postprocedural management protocols are not completely established, even though a small patient series show promising results. ${ }^{5,6}$
Venous sinus stenosis needs to be considered in the differential workup of isolated PT, namely, when the characteristics of the tinnitus suggest a venous origin. Careful evaluation of the venous sinuses using angiographic methods may reveal inconspicuous stenosis, and endovascular treatment with stenting may be considered in selected cases.

\section{ETHICS INFORMATION}

This study complies with the Declaration of Helsinki and was conducted in accordance to the local ethics committee requirements. No figures or videos contain information which allows patient identification; therefore, written patient consent for the publication was not obtained.

\section{Authors' Disclosures}

None.

\section{Statement of Authorship}

The acquisition of data, clinical and imaging data review, literature review, and final manuscript writing were done by MQN. The clinical data review and literature review were done by EF. The acquisition of data, imaging data review, and literature review were done by JMA. The imaging data review and important intellectual contribution were done by JR. The clinical data review, literature review, and final manuscript writing were done by JP.

\section{REFERENCES}

1. Hofmann E, Behr R, Neumann-Haefelin T, Schwager K. Pulsatile tinnitus: imaging and differential diagnosis. Dtsch Arztebl Int. 2013;110(26):451-8.

2. Levine A, Oron Y. Tinnitus. Handb Clin Neurol. 2015;129:409-31.

3. Russel EJ, Michaelis BJ, Wiet R, Meyer J. Objective pulsesynchronous "essential" tinnitus due to narrowing of the transverse dural venous sinus. Int Tinnitus J. 1995;1:127-37.

From the Neuroradiology Department, Hospital de Braga, Braga, Portugal (MQN, JMA, JR); Neurology Department, Hospital de Santa Luzia, Unidade Local de Saúde do Alto Minho, Viana do Castelo, Portugal (EF); Department of Neurology, RWTH Aachen University, Aachen, Germany (JP)

Received February 18, 2019. Final Revisions Submitted April 2, 2019. Date of Acceptance May 6, 2019.

Correspondence to: Miguel Quintas-Neves, Neuroradiology Department, Hospital de Braga, Sete Fontes, S. Vítor, 4710-243 Braga, Portugal. Email: mlqneves@gmail.com 

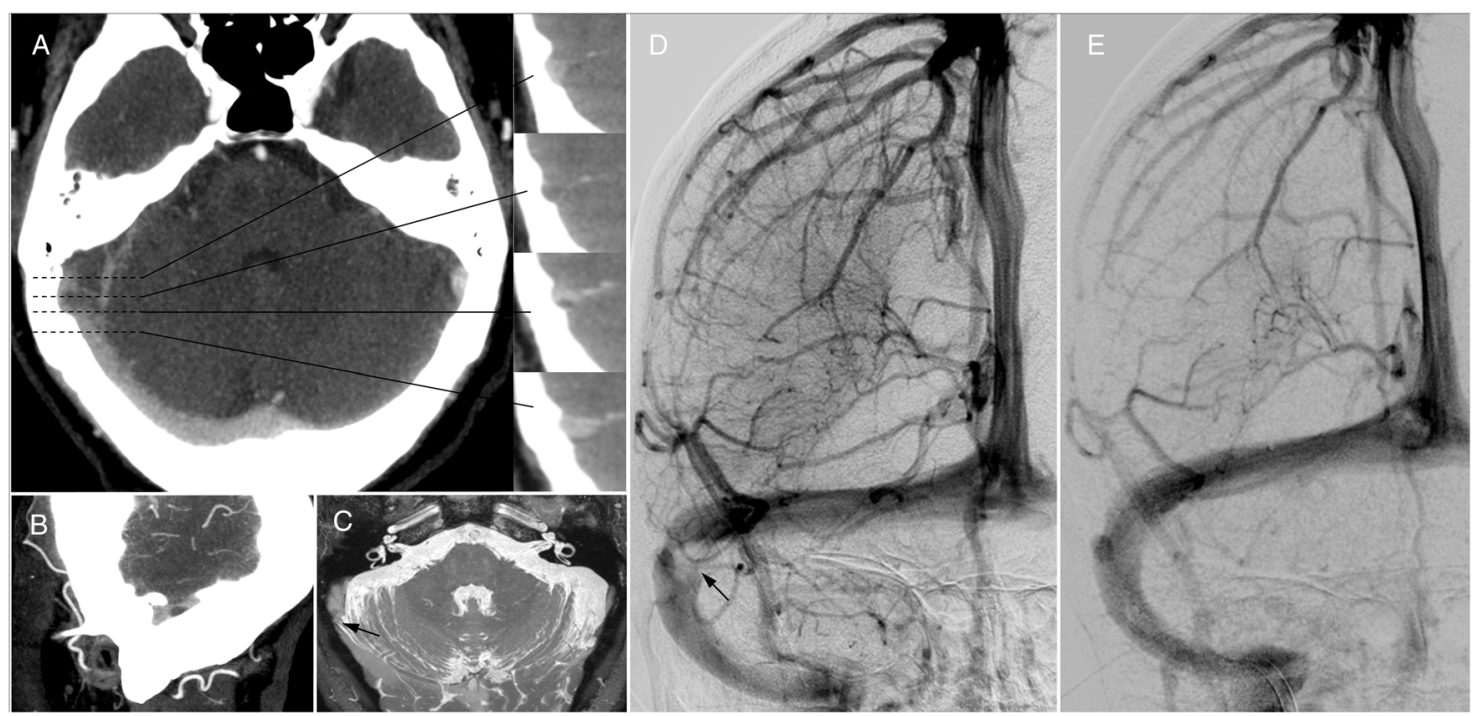

Figure 1: Computed tomography angiography shows a right lateral sinus stenosis in axial, coronal (A) and sagittal (B) reconstructions, caused by a probable Pacchioni's arachnoid granulation. Magnetic resonance imaging (CISS sequence) also shows the right lateral sinus stenosis $(C$, arrow). Digital subtraction angiography confirms a stenosis in the right lateral venous sinus (D, arrow), where a pressure gradient of $9 \mathrm{mmHg}$ was quantified, and normal sinus calibre re-establishment after stenting, with no residual pressure gradient $(E)$.

4. Nicholson P, Brinjikji W, Radovanovic I, et al. Venous sinus stenting for idiopathic intracranial hypertension: a systematic review and meta-analysis. J Neurointerv Surg. 2019;11(4):380-5.

5. Baomin L, Yongbing S, Xiangyu C. Angioplasty and stenting for intractable pulsatile tinnitus caused by dural venous sinus stenosis: a case series report. Otol Neurotol. 2014;35: 366-70.

6. Levitt MR, Albuquerque FC, Gross BA, et al. Venous sinus stenting in patients without idiopathic intracranial hypertension. J Neurointerv Surg. 2017;9(5):512-5. 